\title{
SPITZER AS A MICROLENS PARALLAX SATELLITE: MASS MEASUREMENT FOR THE OGLE-2014-BLG-0124L PLANET AND ITS HOST STAR
}

\author{
A. Udalski ${ }^{1}$, J. C. Yee ${ }^{2,11}$, A. Gould ${ }^{3}$, S. CArey ${ }^{4}$, W. Zhu ${ }^{3}$, J. Skowron ${ }^{1}$, S. KozŁowski ${ }^{1}$, R. Poleski ${ }^{1,3}$, P. Pietrukowicz ${ }^{1}$, \\ G. Pietrzyński ${ }^{1,5}$, M. K. Szymański ${ }^{1}$, P. Mróz ${ }^{1}$, I. Soszyński ${ }^{1}$, K. UlaczyK ${ }^{1}$, Ł. WyrzyKowski $^{1,6}$,

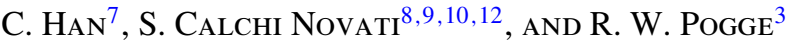 \\ ${ }^{1}$ Warsaw University Observatory, Al. Ujazdowskie 4, 00-478 Warszawa, Poland \\ ${ }^{2}$ Harvard-Smithsonian Center for Astrophysics, 60 Garden St., Cambridge, MA 02138, USA \\ ${ }^{3}$ Department of Astronomy, Ohio State University, 140 W. 18th Ave., Columbus, OH 43210, USA \\ ${ }^{4}$ Spitzer Science Center, MS 220-6, California Institute of Technology, Pasadena, CA, USA \\ ${ }^{5}$ Universidad de Concepción, Departamento de Astronomia, Casilla 160-C, Concepción, Chile \\ ${ }^{6}$ Institute of Astronomy, University of Cambridge, Madingley Road, Cambridge CB3 OHA, UK \\ ${ }^{7}$ Department of Physics, Chungbuk National University, Cheongju 371-763, Korea \\ ${ }^{8}$ NASA Exoplanet Science Institute, MS 100-22, California Institute of Technology, Pasadena, CA 91125, USA \\ ${ }^{9}$ Dipartimento di Fisica "E. R. Caianiello," Università di Salerno, Via Giovanni Paolo II, 84084 Fisciano (SA), Italy \\ ${ }^{10}$ Istituto Internazionale per gli Alti Studi Scientifici (IIASS), Via G. Pellegrino 19, 84019 Vietri Sul Mare (SA), Italy \\ Received 2014 October 23; accepted 2014 December 9; published 2015 February 2
}

\begin{abstract}
We combine Spitzer and ground-based observations to measure the microlens parallax vector $\boldsymbol{\pi}_{\mathrm{E}}$, and thus the mass and distance of OGLE-2014-BLG-0124L, making it the first microlensing planetary system with a space-based parallax measurement. The planet and star have masses of $m \sim 0.5 M_{\text {jup }}$ and $M \sim 0.7 M_{\odot}$ and are separated by $a_{\perp} \sim 3.1 \mathrm{AU}$ in projection. The main source of uncertainty in all of these numbers (approximately 30\%, 30\%, and $20 \%$ ) is the relatively poor measurement of the Einstein radius $\theta_{\mathrm{E}}$, rather than uncertainty in $\pi_{\mathrm{E}}$, which is measured with $2.5 \%$ precision. This compares to $22 \%$ based on OGLE data alone, implying that the Spitzer data provide not only a substantial improvement in the precision of the $\pi_{\mathrm{E}}$ measurement, but also the first independent test of a ground-based $\boldsymbol{\pi}_{\mathrm{E}}$ measurement.
\end{abstract}

Key words: gravitational lensing: micro - planetary systems

\section{INTRODUCTION}

Observing microlensing events from a "parallax satellite" is a powerful way to constrain or measure the lens mass, as was first suggested a half century ago by Refsdal (1966). This idea has acquired increased importance as microlensing planet searches have gained momentum, since obtaining masses and distances for these systems is the biggest challenge facing the microlensing technique. By chance, the typical scale of Galactic microlensing events is $\mathcal{O}(\mathrm{AU})$, which is why it is a good method to find extrasolar planets (Gould \& Loeb 1992). By the same token, a microlensing satellite must be in solar orbit in order for its parallax observations (combined with those from Earth) to probe this distance scale. Hence, it was long recognized that the Spitzer spacecraft, using the $3.6 \mu \mathrm{m}$ channel on its IRAC camera would make an excellent microlensing parallax satellite (Gould 1999).

Nevertheless, until this year, Spitzer had made only one microlensing parallax measurement, which was of an event with a serendipitously bright source star in the Small Magellanic Cloud, OGLE-2005-SMC-001 (Dong et al. 2007). In 2014, however, we received $100 \mathrm{hr}$ of observing time to carry out a pilot program of microlens parallax observations toward the Galactic bulge, with the primary aim of characterizing planetary events.

Here, we report on the first result from that program, a mass and distance measurement for the planet OGLE-2014-BLG$0124 \mathrm{Lb}$

The microlens parallax $\pi_{\mathrm{E}}$ is a two-dimensional vector defined by

$$
\boldsymbol{\pi}_{\mathrm{E}} \equiv \frac{\pi_{\mathrm{rel}}}{\theta_{\mathrm{E}}} \frac{\boldsymbol{\mu}}{\mu} .
$$

\footnotetext{
${ }^{11}$ Sagan Fellow.

${ }^{12}$ Sagan Visiting Fellow.
}

This relation was originally written down by Gould (2000b) with his introduction of the natural formalism for microlensing. For a didactic explanation of how microlensing parallax works, the reader is referred to Figure 1 of Gould \& Horne (2013).

The magnitude of $\pi_{\mathrm{E}}$ vector is the lens-source relative parallax $\pi_{\text {rel }}\left(\pi_{\text {rel }}=\pi_{L}-\pi_{S}=\mathrm{AU}\left(D_{\mathrm{L}}^{-1}-D_{\mathrm{S}}^{-1}\right)\right)$ scaled to the Einstein radius $\theta_{\mathrm{E}}$. This is because $\pi_{\text {rel }}$ determines how much the lens and source will be displaced in angular separation as the observer changes location, while $\theta_{\mathrm{E}}$ sets the angular scale of microlensing phenomena, i.e., the mapping of the physical effect of the displacement onto the light curve. The direction of $\pi_{\mathrm{E}}$ is the same as that of the lens-source relative proper motion $\mu$ because this direction determines how the lens-source displacement will evolve with time.

Combining Equation (1) with the definition of $\theta_{\mathrm{E}}$,

$$
\theta_{\mathrm{E}} \equiv \sqrt{\kappa M \pi_{\mathrm{rel}}} ; \quad \kappa \equiv \frac{4 G}{c^{2} \mathrm{AU}} \simeq 8.14 \frac{\mathrm{mas}}{M_{\odot}},
$$

yields a solution for the lens mass $M$

$$
M=\frac{\theta_{\mathrm{E}}}{\kappa \pi_{\mathrm{E}}}=\frac{\mu t_{\mathrm{E}}}{\kappa \pi_{\mathrm{E}}} .
$$

Hence, if $\pi_{\mathrm{E}}$ and $\theta_{\mathrm{E}}$ are both measured, the mass is determined from the first form of this equation. However, even if $\theta_{\mathrm{E}}$ is not measured, the second form of Equation (3) gives a good estimate of the mass because $t_{\mathrm{E}}$ is almost always known quite well and the great majority of microlensing events will have proper motions within a factor of two of $\mu \sim 4$ mas $\mathrm{yr}^{-1}$. By contrast, if neither $\theta_{\mathrm{E}}$ nor $\pi_{\mathrm{E}}$ is measured, a mass estimate based on $t_{\mathrm{E}}$ alone is extremely crude. See Figure 1 from Gould (2000a).

Since $\pi_{\mathrm{E}}^{2}=\pi_{\text {rel }} / \kappa M$, typical values are $\pi_{\mathrm{E}} \sim 0.3$ for lenses in the Galactic disk and $\pi_{\mathrm{E}} \sim 0.03$ for lenses in the Galactic bulge. 


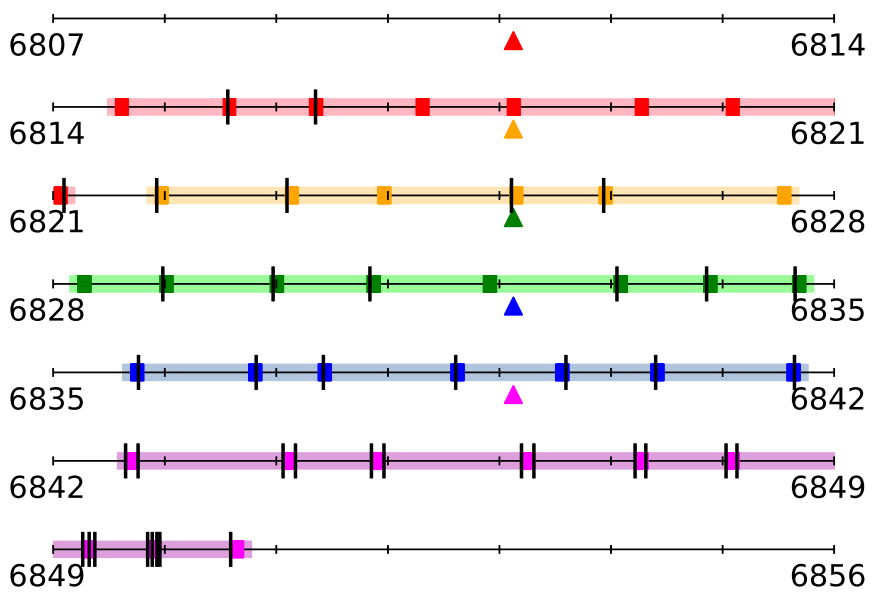

Figure 1. Timeline $\left(\mathrm{HJD}^{\prime}\right)$ of Spitzer observations. Each Spitzer "week" of observations is color-coded. The triangle indicates the date the microlensing targets were submitted to the Spitzer Science Center for observations during the corresponding "week" indicated by the light bands. The solid sections of the bands indicate the blocks allocated to microlensing observations, which were taken approximately once per day. The black, vertical lines indicate the specific observations of OGLE-2014-BLG-0124. These observations were more sparse early in the Spitzer campaign and became more dense as the event neared peak (as seen from Earth) and was discovered to host a planet.

Hence, the projected Einstein radius $\tilde{r}_{\mathrm{E}} \equiv \mathrm{AU} / \pi_{\mathrm{E}}$ typically lies in the range from one AU to several tens of AU. Thus, to see a substantially different event from that seen from Earth requires the satellite to be in solar orbit.

\section{OBSERVATIONS}

We combine observations from two observatories, Spitzer and the Optical Gravitational Lensing Experiment (OGLE).

\subsection{Spitzer Program}

The Spitzer observations were carried out under a $100 \mathrm{hr}$ pilot program granted by the Director to determine the feasibility of Spitzer microlens parallax observations toward the Galactic bulge. Due to Sun-angle viewing constraints, targets near the ecliptic (including bulge microlensing fields) are observable for two $\sim 38$ day continuous viewing periods per 372 day orbital period. Our observation period (2014 June 6 to July 12) was chosen to maximize observability of likely targets, which are grouped in a relatively narrow range of right ascension near $18.0 \mathrm{hr}^{13}$ Targets were observed during $382.63 \mathrm{hr}$ epochs, separated by roughly one day, from $\mathrm{HJD}^{\prime}=\mathrm{HJD}-2450000=$ 6814.0 to 6850.0 .

Each observation consisted of six dithered $30 \mathrm{~s}$ exposures in a fixed pattern using the $3.6 \mu \mathrm{m}$ channel on IRAC. Taking account of various overheads, including time to slew to new targets, this permitted observation of about 34 targets per epoch. ${ }^{14}$

The process of choosing targets and the cadence at which they were observed was complex. A special observing mode was developed specifically for this project. The $382.6 \mathrm{hr}$ epochs were set aside in the Spitzer schedule well in advance. Then, each Monday at UT 15:00, draft sequences were uploaded to Spitzer

\footnotetext{
13 Note that although these targets are equally visible from Spitzer during an interval that is 186 days later, they would be behind the Sun as viewed from Earth, making parallax measurements impossible.

14 Note that the slew time for this program is significantly shorter than is typical for Spitzer because the targets are grouped within a few degrees of each other on the sky.
}

operations for observations to be carried out from Thursday to Wednesday (with some slight variations). These sequences were then vetted for suitability, primarily Sun-angle constraints, and then uploaded to the spacecraft.

Thus, the first problem was to identify targets that could usefully be observed three to nine days in advance of the actual observations. The first reason that this is challenging is that it is usually difficult to predict the evolution of a microlensing light curve from the rising wing, particularly at times well before peak. The characteristic timescale of microlensing events is $t_{\mathrm{E}} \sim 25$ days. Hence, for example, nine days before peak an event with a typical source magnitude $I_{s}=19$ would be only 1 mag brighter, $I=18$, meaning that ground-based photometry would be relatively poor, allowing only a crude prediction of its evolution. Such predictions are typically consistent with a broad range of fits, extending from the event peaking not much brighter than its current brightness (implying it would be unobservably faint in Spitzer data) to peaking at very high magnification (which would allow an unambiguous Spitzer parallax measurement). The second reason is that Spitzer will necessarily see a different light curve than the one from the ground (this is the point of parallax observations!). Observations are much more likely to yield good parallax measurements if the event peaks as seen by Spitzer, but depending on the value of the parallax, this peak could be very similar to the peak time seen from Earth or days or weeks earlier or later.

To address the first challenge, J.C.Y. wrote software to automatically fit all ongoing microlensing events and assess whether or not they met criteria for inclusion in the Spitzer observation campaign. This software is based on a code written by A.G. and J.C.Y. that has been used successfully for many years to predict high-magnification events. It was tested on OGLE data from the 2013 microlensing season and used to simulate the Spitzer observations by fitting the data for each event up to a certain cutoff date, and repeating for successive weeks. Then J.C.Y. and A.G. estimated the correctness of these automated choices by comparing to fits of the complete light curves, that is, determining whether or not an event classification based on incomplete data was correct when compared to the final, known properties of that event. This served as the basis both for fine-tuning the software and for learning when to manually override it. These lessons were then applied each week by J.C.Y. and A.G. to the actual choice of targets.

To further expedite this process, OGLE set up a special realtime reduction pipeline for potential targets under consideration, with updates lagging observations by just a few minutes. This permitted robust construction of a trial protocol at about UT 03:00 on Monday, and late-time tweaking based on the most recent OGLE data (typically ending at UT 10:00) for final internal vetting and translation into a set of "Astronomical Observation Requests" before uploading to Spitzer operations.

The next problem was to determine the cadence. The program limited observations to $2.6 \mathrm{hr}$ windows roughly once per day. This precluded using Spitzer to find planets, since this requires observations at several-to-many times per day. Moreover, since there were usually more than 34 targets that could usefully be observed during a given week, not all of the targets could be observed at every epoch. Targets were thus divided into "daily," "moderate," and "low" cadence. The first were observed every epoch, the second were observed most epochs, and the third were observed about one-third to one-half of the epochs. In addition, a few targets were regarded as "very high priority" and thus were slated to be observed more than once per epoch. 


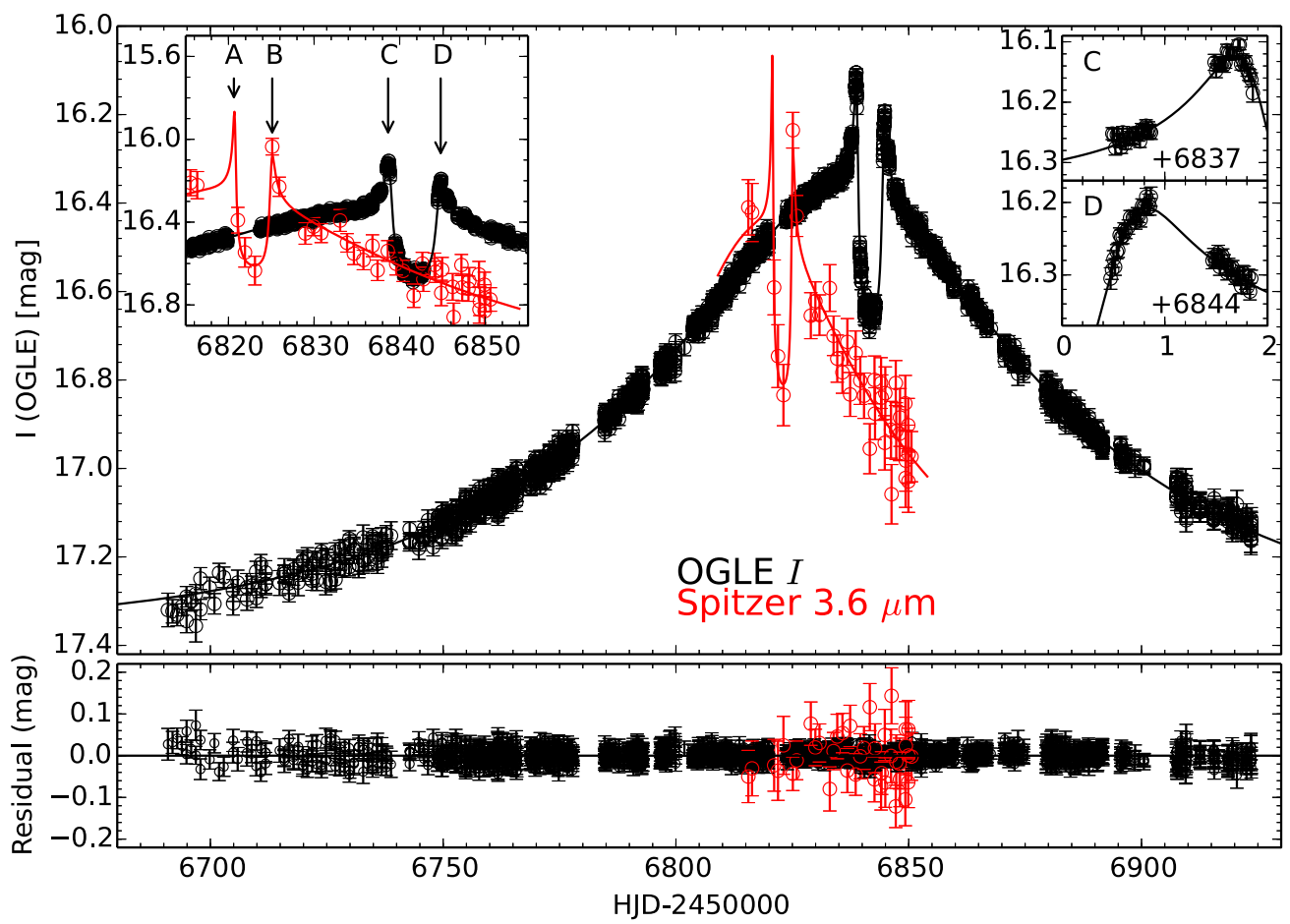

Figure 2. Light curve and residuals for planetary model of OGLE-2014-BLG-0124 as observed from Earth by OGLE in $I$ band (black) and by Spitzer at $3.6 \mu \mathrm{m}$ (red), which was located $\sim 1 \mathrm{AU}$ west of Earth in projection at the time of the observations. A simple inspection of the OGLE light curve features shows that this is Jovian planet, while the fact that Spitzer observed similar features 20 days earlier demonstrates that the lens is moving $\tilde{v} \sim 105 \mathrm{~km} \mathrm{~s}^{-1}$ due east projected on the plane of the sky (Section 3). Detailed model-fitting confirms and refines this by-eye analysis (Section 5). Note that in the left inset, the Spitzer light curve is aligned to the OGLE system (as is customary), but it is displaced by $0.2 \mathrm{mag}$ in the main diagram, for clarity.

Particularly during the first week, when there were many targets that had just peaked (and, of course, had not yet been observed), targets that were predicted for peak many weeks in the future were downgraded in priority. This constraint directly impacted observations of OGLE-2014-BLG-0124.

\subsection{OGLE Observations}

On 2014 February 22 OGLE alerted the community of a new microlensing event OGLE-2014-BLG-0124 based on observations with the $1.4 \mathrm{deg}^{2}$ camera on its $1.3 \mathrm{~m}$ Warsaw Telescope at the Las Campanas Observatory in Chile using its Early Warning System (EWS) real-time event detection software (Udalski et al. 1994; Udalski 2003). Most observations were in $I$ band, with a total of $20 \mathrm{~V}$-band observations during 2014 to determine the source color. The source star lies at (R.A., decl. $)=(18: 02: 29.21,-28: 23: 46.5)$ in OGLE field BLG512, which is observed at OGLE's highest cadence, about once every 20 minutes.

On June 29 UT 17:05, our group alerted the microlensing community to an anomaly in this event, at that time of unknown nature, based on the analysis of OGLE data from the special pipeline described above. While in some cases (e.g., Yee et al. 2012) OGLE responds to such alerts by increasing its cadence, it did not do so in this case because of the high cadence already assigned to this field. Hence, OGLE observations are exactly what they would have been if the anomaly had not been noticed.

For the final analysis, the OGLE data set was re-reduced. Optimal photometry was derived with the standard OGLE photometric pipeline (Udalski 2003) tuned-up to the OGLEIV observing set-up, after deriving an accurate centroid of the source star.

\subsection{Spitzer Cadence}

At the decision time (June 2 UT 15:00, HJD' 6811.1) for the first week of Spitzer observations, OGLE-2014-BLG-0124 was regarded as a promising target, but because it appeared to be peaking 30-40 days in the future, as predicted by the event selection software described in Section 2.1, it was assigned "moderate" priority, which implied that it was observed in only three of the first eight epochs because of the large number of targets in the first week. The following week, it was degraded to "low" priority because its estimated peak receded roughly one week into the future. Nevertheless, because the total number of targets fell from 44 to 37, OGLE-2014-BLG-0124 was observed during four of the six epochs scheduled that week. Since the peak was approaching, it was raised back to "moderate" priority in the third week and observed in six out of eight epochs, and then to "daily" priority in the fourth week and observed in all seven epochs. It was the review of events in preparation for the fifth week that led to the recognition that OGLE-2014-BLG-0124 was undergoing an anomaly (Section 2.2), and hence it was placed at top priority. In addition, as the week proceeded, the events lying toward the west of the microlensing field gradually moved beyond the allowed Sun-angle range, which permitted more observations of those (like OGLE-2014-BLG-0124) that lay relatively to the east. As a result, it was observed a total of 20 times in eight epochs.

In fact, due to the particular configuration of the event, the most crucial observations turned out to be those during the first 10 days when the event was rated as "low" to "moderate" priority. See Figures 1 and 2.

The Spitzer data were reduced using the DoPhot pointspread function (PSF) photometry program (Schechter et al. 1993) after experimentation with a few software packages 


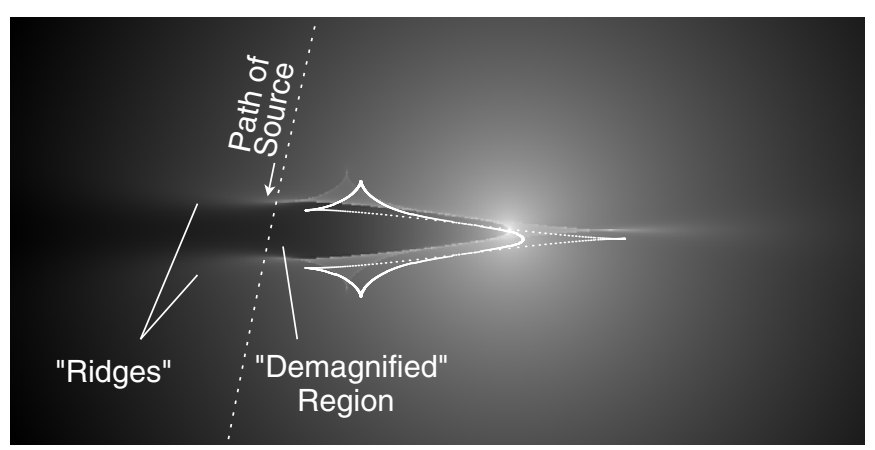

Figure 3. Magnification map for the caustic region of OGLE-2014-BLG-0124 in standard orientation with the planet to the right. As the source passes over the "demagnified" region (darker tones), the minor image due (to the primary lens) passes very close to the planet, which is off the figure to the right. Because the minor image is unstable, it is easily destroyed by the planet, which accounts for the relative demagnification. Two triangular caustic regions flank the deepest part of this demagnification. The source does not cross these caustics, but does cross the two ridges that extend from the cusps, toward the left. It is these ridges that are responsible for the two bumps near $t=6820$ and $t=6825$ (from Spitzer) or $t=6839$ and $t=6845$ (from Earth) in Figure 2.

(e.g., the standard Spitzer PSF/PSR software, image subtraction technique). DoPhot's superior performance in the case of the blended event OGLE-2014-BLG-0124 may be related to its capability of holding the source position (photometry centroid) fixed in a series of images and the fact that the OGLE-2014-BLG-0124 image is isolated on scales of the Spitzer PSF.

\section{HEURISTIC ANALYSIS}

The most prominent feature in the OGLE light curve (black points, Figure 2) is a strong dip very near what would otherwise be the peak of the light curve (HJD' $\sim 6842$ ). The dip is flanked by two peaks (highlighted in the insets), each of which is pronounced, but neither of which displays the violent breaks characteristic of caustic crossings. This dip must be due to an interaction between a planet and the minor image created by the host star in the underlying microlensing event. That is, in the absence of a planet, the host will break the source light into two magnified images, a major image outside the Einstein ring on the same side as the source and a minor image inside the Einstein ring on the opposite side from the source (e.g., Gaudi 2012). Being at a saddle point of the time-delay surface, the minor image is highly unstable to perturbations, and is virtually annihilated if a planet lies in or very near its path. These (relatively) demagnified regions are always flanked by two triangular caustics (see Figure 3 ). If the source had passed over these caustics, it would have shown a sharp break in the light curve because the magnification of a point source diverges to infinity as it approaches a caustic. Hence, from the form of the perturbation, it is clear that the source passed close to these caustics but not directly over them. Because the two peaks are of nearly equal height, the source passed so the angle $\alpha$ between its path and planet-star axis is roughly $90^{\circ}$.

The Spitzer light curve (red points, Figure 2) shows very similar morphological features but displaced about 19.5 days earlier in time. The velocity of the lens relative to the source (projected onto the observer plane) $\tilde{\mathbf{v}}$ is easily measured by combining information from the Spitzer light curve and the OGLE light curve. This is the most robustly measured quantity derived from the light curve, and it is related to the parallax vector by

$$
\boldsymbol{\pi}_{\mathrm{E}}=\frac{\mathrm{AU}}{t_{\mathrm{E}}} \frac{\tilde{\mathbf{v}}}{\tilde{v}^{2}} .
$$

Projected on the plane of the sky, Spitzer's position at the time it saw the dip (HJD' 6822.5) was about 1.17 AU away from where the Earth was when it saw the dip (HJD' 6842), basically due west of Earth. Hence, the projected velocity of the lens relative to the source (in the heliocentric frame) along this direction is $\tilde{v}_{\text {hel }, E} \sim 1.17 \mathrm{AU} /(19.5$ day $) \sim 105 \mathrm{~km} \mathrm{~s}^{-1}$. On the other hand, the fact that the morphology is similar shows that the source passed the caustic structure at a similar impact parameter perpendicular to its trajectory (i.e., in the north direction). Hence $\tilde{v}_{\text {hel }, N} \sim 0$. One converts from heliocentric to geocentric frames by $\tilde{\mathbf{v}}_{\text {hel }}=\tilde{\mathbf{v}}_{\text {geo }}+\mathbf{v}_{\oplus, \perp}$ where $\mathbf{v}_{\oplus, \perp}(\mathrm{N}, \mathrm{E}) \simeq(0,30) \mathrm{km} \mathrm{s}^{-1}$ is the velocity of Earth projected on the sky at the peak of the event. Hence,

$$
\begin{aligned}
\tilde{\mathbf{v}}_{\text {hel }} & =\tilde{\mathbf{v}}_{\text {geo }}+\mathbf{v}_{\oplus, \perp} \simeq(0,105) \mathrm{km} \mathrm{s}^{-1} . \\
\tilde{\mathbf{v}}_{\text {geo }}(\mathrm{N}, \mathrm{E}) & \simeq(0,75) \mathrm{km} \mathrm{s}^{-1} ;
\end{aligned}
$$

This result is robust and does not depend in any way on the details of the analysis.

Next, we estimate the planet-host mass ratio $q$ and the planethost projected separation $s$ in units of the Einstein radius making use of three noteworthy facts. First, because the perturbation affects the minor image, $s<1$. Second, by making the approximation that the planet passes directly over the minor image, we can express the position of the source as $u=1 / s-s$. Third, because the perturbation occurs close to the time of the peak, $u_{\text {perturbation }} \simeq u_{0}$, i.e., $u_{0} \simeq 1 / s-s$.

The impact parameter between the source and lens $u_{0}$ can be estimated from the peak magnification of the event $A_{\max }$. As we show immediately below, the source star is significantly blended with another star or stars that lie within the PSF, but that do not participate in the event. Nevertheless, for simplicity of exposition, we initially assume that the source is not blended and then subsequently incorporate blending into the analysis. Under this assumption, the fact that the peak of the underlying point-lens event is a magnitude brighter than baseline implies a peak magnification $A_{\max }^{\text {naive }}=2.5$ and thus an impact parameter $u_{0}^{\text {naive }} \sim 1 / A_{\max }^{\text {naive }} \sim 0.40$ and $s \sim 0.82$. Additionally, the fact that the event becomes a factor 1.34 brighter (corresponding to entering $u=1$ ) roughly 60 days before peak, implies $t_{\mathrm{E}}^{\text {naive }}=60$ days.

We can now make use of the analytic estimate of Han (2006) for the perpendicular separation $\eta_{c,-}$ (normalized to $\theta_{\mathrm{E}}$ ) between the planet-star axis and the inner edge of the triangular planetary caustic due to a minor-image perturbation (see Figure 2 of Han 2006),

$$
\eta_{c,-}^{2} \simeq 4 \frac{q}{s}\left(\frac{1}{s}-s\right)
$$

to estimate $q$. Because the source passes nearly perpendicular to the planet-star axis, we have $\eta_{c,-} \simeq \Delta t_{c,-} / t_{\mathrm{E}}$, where $\Delta t_{c,-}=3$ days is half the time interval between the two peaks. Then solving for $q$ yields

$$
\begin{aligned}
q & =\frac{s}{4 u_{0}}\left(\frac{\Delta t_{c,-}}{t_{\mathrm{E}}}\right)^{2}=\frac{s}{4} \frac{\Delta t_{c,-}^{2}}{t_{\mathrm{E}} t_{\mathrm{eff}}} \\
& =1.56 \times 10^{-3} s\left(\frac{t_{\mathrm{E}}}{60 \text { day }}\right)^{-1}\left(\frac{t_{\mathrm{eff}}}{24 \text { day }}\right)^{-1},
\end{aligned}
$$

where $t_{\text {eff }} \equiv u_{0} t_{\mathrm{E}}$ is the effective timescale. Now, whereas $t_{\mathrm{E}}$ is very sensitive to blending (because a fainter source requires 
higher magnification-so further into the Einstein ring-to achieve a given increase in flux), $t_{\text {eff }}$ is not. In addition, $u_{0}<u_{0}^{\text {naive }}=0.40$ implies $s>0.82$, i.e., close to unity in any case. Thus to first order, $q$ is inversely proportional to $t_{\mathrm{E}}$. This implies a Jovian mass ratio unless the blended flux were many times higher than the source flux, in which case the mass ratio would be substantially lower.

Finally, we note that the absolute position of the source, which can be determined very precisely on difference images because the source is then isolated from all blends, is displaced from the naive "baseline object" by 80 mas. Additionally, given that the source and blend are not visibly separable in the best seeing images, they must be closer than 800 mas. The combination of these facts means that the blend must contribute at least $10 \%$ of the light. However, precise determination of the blending requires detailed modeling, to which we now turn.

\section{LIGHT CURVE ANALYSIS}

In addition to the parameters mentioned in the previous section $\left(u_{0}, t_{\mathrm{E}}, q, s, \alpha, \pi_{\mathrm{E}}\right)$ and $t_{0}$ (where $t=t_{0}$ at $\left.u=u_{0}\right)$, we include three additional parameters in the modeling. The first is $\rho \equiv \theta_{*} / \theta_{\mathrm{E}}$, where $\theta_{*}$ is the angular radius of the source star. This is closely related to the source radius self-crossing time, $t_{*} \equiv \rho t_{\mathrm{E}}$. Any sharp breaks in the underlying magnification pattern will be smoothed out on the scale of $t_{*}$, which is how it is normally measured. In fact, there are no such sharp breaks because there are no caustic crossings. However, the ridges of magnification seen in Figure 3 that give rise to the two bumps near the peak of the light curve are relatively sharp and thus may be sensitive to $\rho$.

Second, we allow for orbital motion of the planet-star system. We consider only two-dimensional motion in the plane of the sky, which we parameterize by $d s / d t$ (a uniform rate of change of planet-host separation) and $d \alpha / d t$ (a uniform rate of change in position angle). Because the orbital period is likely to be of the order of several years while the baseline of measurement between caustic features seen in the Earth and Spitzer light curves is only about 22 days, we do not expect to have sensitivity to additional parameters. In fact, we will see that even one of these two orbital parameters is poorly constrained; thus, there is no basis to include additional ones.

Therefore, there are 11 model parameters $\left(t_{0}, u_{0}, t_{\mathrm{E}}, \rho, \pi_{\mathrm{E}, E}\right.$, $\left.\pi_{\mathrm{E}, \mathrm{N}}, s, q, \alpha, d s / d t, d \alpha / d t\right)$, plus two flux parameters $\left(f_{s}, f_{b}\right)$ for each observatory. For completeness, we specify the sign conventions for $u_{0}, \alpha$, and $d \alpha / d t$. We designate $u_{0}>0$ if the moving lens passes the source on its right. We designate $\alpha$ as the (counterclockwise) angle made by the star-to-planet axis relative to the lens-source relative proper motion at the fiducial time, which we choose to be $t_{0, \mathrm{par}}=6842$ (see below). We designate $d \alpha / d t$ to be positive if the projected orbit of the planet is counterclockwise.

We adopt limb darkening coefficients $u_{V}, u_{I}=(0.68,0.53)$ corresponding to $\left(\Gamma_{V}, \Gamma_{I}\right)=(0.59,0.43)$ based on the models of Claret (2000) and the source characterization described in Section 5. For the Spitzer $3.6 \mu \mathrm{m}$ band we adopt $u_{3.6}=0.22$, and so $\Gamma_{3.6}=0.16$, which we extrapolate from the long-wavelength values calculated by Claret (2000).

As is customary, we conduct the modeling in the geocentric frame (defined as the moving frame of Earth at $t_{0, \mathrm{par}}=6842$ ). This time is close to the midpoint of the two cusp-approaches observed by OGLE (see Figure 2), which is when the angular orientation of the planet-host system is best defined (and so has the smallest formal error). It may seem more natural to use the heliocentric frame, given that we have observations from two different heliocentric platforms. However, we adopt the geocentric frame for two reasons. First, this permits the simplest comparison to results derived without Spitzer data. Second, the geocentric computational formalism is well established, so keeping it minimizes the chance of error. From an algorithmic point of view, Spitzer's orbital motion is incorporated as a standin for the usual "terrestrial parallax" term. That is, whereas other observatories are displaced from Earth's center according to their location and the sidereal time, Spitzer is displaced from Earth's center according to its tabulated distance and position on the sky as seen from Earth. In this way, our approach takes into account both Spitzer's mean offset from Earth and its motion relative to Earth during the observations.

As usual, we use the point source approximation for epochs that are far from the caustics and the hexadecapole approximation (Pejcha \& Heyrovský 2009; Gould 2008) at intermediate distances. For epochs that are near or on crossing caustics, we use contour integration (Gould \& Gaucherel 1997). In practice, contour integration is not needed at all for the ground-based data and is used for only five of the Spitzer data points, i.e., those that might conceivably pass close to a caustic. To accommodate limb darkening, we divide the surface into 10 annuli, although this is severe overkill in Spitzer's case because of its low value of $\Gamma=0.16$.

We both search for the minimum and find the likelihood distribution of parameter combinations using a Markov Chain Monte Carlo (MCMC).

\subsection{Estimate of $\theta_{*}$}

Before discussing the model parameters, we focus first on the flux parameters, which enable a determination of $\theta_{*}$. Based on calibrated OGLE magnitudes, we find $f_{s, \text { ogle }}=0.579 \pm 0.013$, $f_{b, \text { ogle }}=1.213 \pm 0.013$, in a system in which $f=1$ corresponds to an $I=18$ star, i.e., $I_{s}=18.59 \pm 0.02, I_{b}=17.79 \pm 0.01$. Using the standard approach (Yoo et al. 2004), we determine the dereddened source brightness $I_{s, 0}=17.57$ from the offset from the red clump using tabulated clump brightness as a function of position from Nataf et al. (2013). Similarly, we determine the apparent $(V-I)_{s}$ color from regression of $V$ and $I$ flux over the event (i.e., without reference to any model), and then find $(V-I)_{0}=0.70$ from the offset to the clump, with assumed intrinsic color of $(V-I)_{0, \mathrm{cl}}=1.06$ (Bensby et al. 2013). We then convert from $(V-I)$ to $(V-K)$ using the empirical color-color relations of Bessell \& Brett (1988) and finally estimate the source radius using the color/surface-brightness relation of Kervella et al. (2004). We find

$$
\theta_{*}=0.95 \pm 0.07 \mu \text { as. }
$$

The error is completely dominated by the $0.05 \mathrm{mag}$ error in the derivation of the intrinsic source color (Bensby et al. 2013), and an adopted $0.1 \mathrm{mag}$ error for vertical centroiding of the clump.

\subsection{Physical Constraints on Two Parameters}

We find that the fits to light curve data leave two parameters poorly constrained: $\rho$ and $d \alpha / d t$. Although both distributions are actually well-confined, in both cases a substantial fraction of the parameter space corresponds to unphysical solutions. This is not in itself worrisome: the requirement of consistency with nature only demands that physical solutions be allowed, not that unphysical solutions be excluded by the data. However, it does 
oblige us to outline the relation between physically allowed and excluded solutions before suppressing the latter.

In the case of $\rho$, there is a well-defined range $0<\rho<0.0025$ that is permitted by the light curve data at the $3 \sigma$ level. The upper bound is due to the sharpness of the peak observed in the OGLE data ( $t \sim 6839$ days) as the source crosses the ridge that extends from the tip of the caustic (Figure 3). That is, larger values of $\rho$ cause the model light curve to be more rounded than the data. The lower bound is strictly enforced by the positivity of stellar radii. However, from a pure light curve perspective, $\rho=0$ solutions are consistent at the $1 \sigma$ level. Nevertheless, arbitrarily low values of $\rho$ are not permitted physically because the lens mass and distance can be expressed,

$$
\begin{aligned}
M & =\frac{\theta_{*} / \kappa \pi_{\mathrm{E}}}{\rho}=1.2 M_{\odot} \frac{6.5 \times 10^{-4}}{\rho} ; \\
\pi_{\mathrm{rel}} & =\frac{\pi_{\mathrm{E}} \theta_{*}}{\rho}=0.21 \operatorname{mas} \frac{6.5 \times 10^{-4}}{\rho} .
\end{aligned}
$$

Since $\pi_{\mathrm{E}}=0.15$ is very well determined from the light curve fits and, as we discussed in Section $4.1, \theta_{*}$ is also well determined, the numerators of both expressions in Equation (9) are also well-determined. Hence, as $\rho$ decreases, both $M$ and $\pi_{\text {rel }}$ increase, i.e., the host gets closer and more luminous, hence brighter. The final expressions show our adopted limit. That is, at $\pi_{\text {rel }}=0.21$ mas $\left(D_{L}=3.1 \mathrm{kpc}\right)$ and even assuming that the host star lay behind all the dust seen toward the source $\left(A_{I}=1.06\right)$, the absolute magnitude of the lens is constrained to be $M_{I, L}>I_{b}-A_{I}-5 \log \left(D_{L} / 10 \mathrm{pc}\right)=4.35$ which is considerably dimmer than any $M=1.2 M_{\odot}$ star. Note that this limit $\left(\rho>6.5 \times 10^{-4}\right)$ is quite consistent with the "best-fit" value of $\rho \sim 10^{-3}$, although as emphasized above, this detection of $\rho>0$ is statistically quite marginal.

Second, at the $3 \sigma$ level, $d \alpha / d t$ is constrained by the light curve to the range $0.5<(d \alpha / d t) \mathrm{yr}<5$. However, sufficiently large values of $d \alpha / d t$ lead to unbound systems. This is quantified by the ratio of projected kinetic to potential energy (Dong et al. 2009),

$$
\beta \equiv\left(\frac{E_{\text {kin }}}{E_{\text {pot }}}\right)_{\perp}=\frac{\kappa M_{\odot}(\mathrm{yr})^{2}}{8 \pi^{2}} \frac{\pi_{\mathrm{E}} s^{3} \gamma^{2}}{\theta_{\mathrm{E}}\left(\pi_{\mathrm{E}}+\pi_{s} / \theta_{\mathrm{E}}\right)^{3}}
$$

where

$$
\boldsymbol{\gamma}=\left(\gamma_{\|}, \gamma_{\perp}\right) \equiv\left(\frac{d s / d t}{s}, \frac{d \alpha}{d t}\right)
$$

In this case, one cannot write the resulting limit in such a simple form as was the case for $\rho$. However, adopting a typical value $\rho=10^{-3}$ (and therefore $\theta_{\mathrm{E}}=0.95$ mas) for illustration, and noting that $\gamma_{\|}$is constrained to a range that renders it irrelevant to this calculation, we obtain $\beta=0.59\left[\gamma_{\perp} \mathrm{yr}\right]^{2}$. Since $\beta>1$ implies an unbound system, values of $\left|\gamma_{\perp}\right|>1.3 \mathrm{yr}^{-1}$ are forbidden. This evaluation strictly applies only for solutions with $\rho=10^{-3}$, but actually it evolves only slowly over the allowed range $0.65<10^{3} \rho<2.5$. Typical values expected for $\beta$ are $0.2-0.6$, which occur for $\left|\gamma_{\perp}\right| \sim 1 \mathrm{yr}^{-1}$. While the best-fit value is $\gamma_{\perp}=2 \mathrm{yr}^{-1}$, values of $\gamma_{\perp} \sim 1 \mathrm{yr}^{-1}$ are disfavored at only $1.5 \sigma$. Hence, we conclude that physically allowed systems are close to the overall $\chi^{2}$ minimum and therefore we are justified

\begin{tabular}{|c|c|c|c|}
\hline Parameter & Unit & $u_{0}>0$ & $u_{0}<0$ \\
\hline \multirow[t]{2}{*}{$\chi^{2} /$ dof } & & 6664 & 6671 \\
\hline & & /6769 & $/ 6769$ \\
\hline \multirow[t]{2}{*}{$t_{0}-6800$} & day & 36.176 & 36.140 \\
\hline & & 0.039 & 0.040 \\
\hline \multirow[t]{2}{*}{$u_{0}$} & & 0.1749 & -0.1778 \\
\hline & & 0.0039 & 0.0032 \\
\hline \multirow[t]{2}{*}{$t_{\mathrm{E}}$} & day & 152.1 & 151.8 \\
\hline & & 2.9 & 2.4 \\
\hline \multirow[t]{2}{*}{$s$} & & 0.9443 & 0.9429 \\
\hline & & 0.0030 & 0.0023 \\
\hline \multirow[t]{2}{*}{$q$} & $10^{-3}$ & 0.694 & 0.705 \\
\hline & & 0.046 & 0.038 \\
\hline \multirow[t]{2}{*}{$\alpha$} & deg & 78.216 & -78.307 \\
\hline & & 0.090 & 0.100 \\
\hline \multirow[t]{2}{*}{$\rho$} & $10^{-3}$ & 1.25 & 1.37 \\
\hline & & 0.38 & 0.42 \\
\hline \multirow[t]{2}{*}{$\pi_{\mathrm{E}, \mathrm{N}}$} & & -0.0055 & 0.0399 \\
\hline & & 0.0048 & 0.0052 \\
\hline \multirow[t]{2}{*}{$\pi_{\mathrm{E}, \mathrm{E}}$} & & 0.1461 & 0.1430 \\
\hline & & 0.0037 & 0.0037 \\
\hline \multirow[t]{2}{*}{$\gamma_{\|}$} & $\mathrm{yr}^{-1}$ & -0.115 & -0.119 \\
\hline & & 0.017 & 0.016 \\
\hline \multirow[t]{2}{*}{$\gamma_{\perp}$} & $\mathrm{yr}^{-1}$ & 0.77 & -0.97 \\
\hline & & 0.53 & 0.45 \\
\hline
\end{tabular}
in imposing physical constraints to obtain our final solution.
Table 1

$\mu$ lens Parameters (Spitzer+OGLE)

\section{PHYSICAL PARAMETERS}

Following the arguments in Section 4.2, we impose the following two constraints,

$$
M<1.2 M_{\odot}, \quad \beta<1,
$$

on output chains from our MCMC to obtain final parameters (Table 1) and physical parameters (Table 2). We also considered using the more tapered prior on $\beta$ introduced by Poleski et al. (2014). However, this did not have a perceptible effect on either the values or the errors reported in Tables 1 and 2. Therefore, we adopted the more conservative constraint in Equation (12).

Our solution indicates a $0.5 M_{\text {jup }}$ planet orbiting a $0.7 M_{\odot}$ star that is $4.1 \mathrm{kpc}$ from the Sun, with a projected separation of $3.1 \mathrm{AU}$. This is very close to being a scaled down version of our own Jupiter, with host mass, planet mass, and physical separation (estimated as $\sqrt{3 / 2}$ larger than projected separation) all reduced by a factor of $\sim 0.6$.

\subsection{Discrete Degeneracies}

In his original paper, Refsdal (1966) already noted that spacebased parallaxes for point-lens events are subject to a four-fold discrete degeneracy. This is because the satellite and Earth observatories each see two "bumps," each with different $t_{0}$ and $u_{0}$, and the parallax is effectively reconstructed from the differences in these quantities

$$
\pi_{\mathrm{E}}=\frac{\mathrm{AU}}{D_{\perp}}\left(\frac{\Delta t_{0}}{t_{\mathrm{E}}}, \Delta u_{0}\right),
$$

where, $\Delta t_{0}=t_{0, \text { sat }}-t_{0, \oplus}, \Delta u_{0}=u_{0 \text {, sat }}-u_{0, \oplus}$, and $\mathbf{D}_{\perp}$ is the projected separation vector of Earth and the satellite, whose 

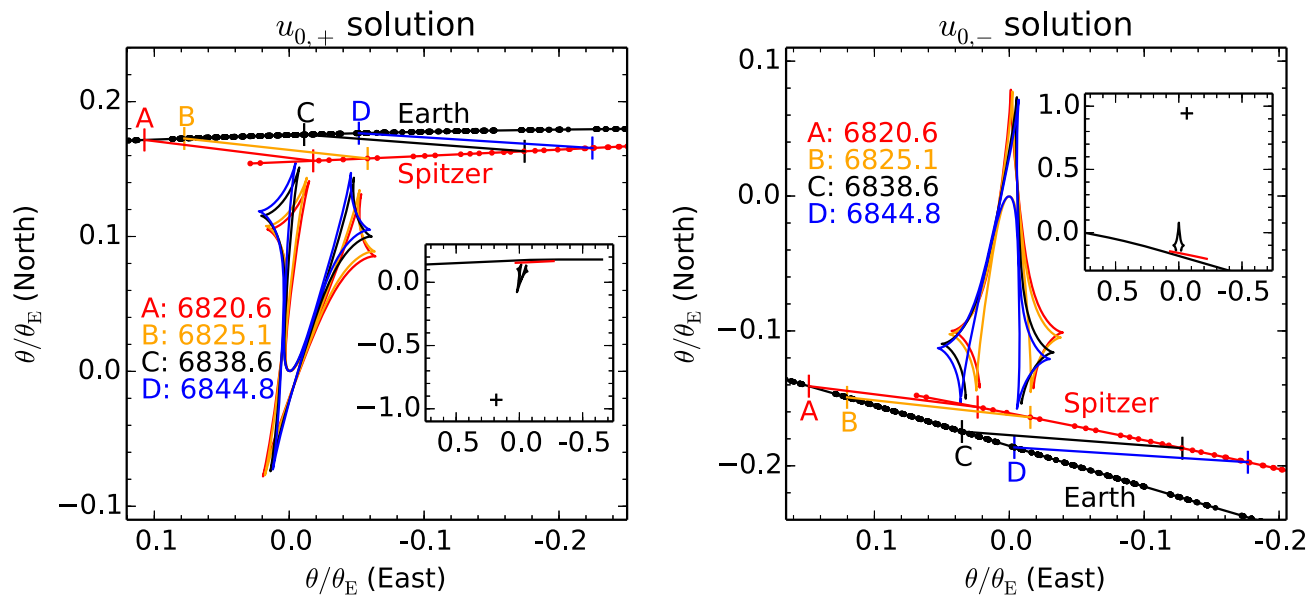

Figure 4. Lens geometry for OGLE-2014-BLG-0124. North is up and east is left. The lens position is fixed, but with its orientation rotating at $d \alpha / d t$ and the planet-star separation changing by $d s / d t$, with the four epochs at which the source passes the two caustic "prongs" as seen from Spitzer and Earth shown in different colors. The source positions as seen from OGLE (black) and Spitzer (red) are shown for each epoch of observation. These trajectories deviate slightly from rectilinear motion because of parallax effects of each observatory's motion. The line segments indicate common times at the two observatories, which illustrate that the Earth-Spitzer projected separation increases substantially over the 35 days of Spitzer observations. The left (right) panel shows the geometry of the $u_{0}>0\left(u_{0}<0\right)$ solutions, which are very similar except for orientation (see Tables 1 and 2). Planet location is indicated by "+" symbols in insets.

Table 2

Physical Parameters (Spitzer+OGLE)

\begin{tabular}{lcrr}
\hline \hline Parameter & Unit & $u_{0}>0$ & $u_{0}<0$ \\
\hline$M_{\text {host }}$ & $M_{\odot}$ & 0.71 & 0.65 \\
& & 0.22 & 0.22 \\
\hline$M_{\text {planet }}$ & $M_{\text {jup }}$ & 0.51 & 0.47 \\
& & 0.16 & 0.15 \\
\hline Distance & $\mathrm{kpc}$ & 4.10 & 4.23 \\
& & 0.59 & 0.59 \\
\hline$a_{\perp}$ & $\mathrm{AU}$ & 3.11 & 2.97 \\
& & 0.49 & 0.51 \\
\hline$\tilde{v}_{\mathrm{N}, \text { hel }}$ & $\mathrm{km} / \mathrm{s}$ & -3.0 & 20.6 \\
& & 2.6 & 2.9 \\
\hline$\tilde{v}_{\mathrm{E}, \text { hel }}$ & $\mathrm{km} / \mathrm{s}$ & 107.0 & 103.2 \\
& & 2.8 & 2.3 \\
\hline$\mu_{\text {hel }}$ & $\mathrm{mas} / \mathrm{yr}$ & 2.77 & 2.56 \\
& & 0.86 & 0.83 \\
\hline$\beta=\left(E_{\text {kin }} / E_{\text {pot }}\right)_{\perp}$ & & 0.47 & 0.57 \\
\hline$\theta_{\mathrm{E}}$ & & 0.29 & 0.30 \\
\hline
\end{tabular}

direction sets the orientation of the $\pi_{\mathrm{E}}$ coordinate system. However, whereas $\Delta t_{0}$ is unambiguously determined from this procedure, $u_{0}$ is actually a signed quantity whose amplitude is recovered from simple point-lens events but whose sign is not. Hence, there are two solutions $\left.\Delta u_{0,-, \pm}= \pm\left(\left|u_{0, \text { sat }}\right|-\mid u_{0, \oplus}\right) \mid\right)$ for which the satellite and Earth observe the source trajectory on the same side of the lens as each other (with the " \pm " designating which side this is), and two others $\left.\Delta u_{0,+, \pm}= \pm\left(\left|u_{0, \text { sat }}\right|+\mid u_{0, \oplus}\right) \mid\right)$ for which the source trajectories are seen on opposite sides of the lens (Gould 1994).

The first of these degeneracies is actually an extension to space-based parallaxes of the $\pm u_{0}$ "constant acceleration degeneracy" for ground-based parallaxes discovered almost $40 \mathrm{yr}$ later by Smith et al. (2003), and which is extended to binary lenses by Skowron et al. (2011). This degeneracy results in a different direction of the parallax vector.
The second degeneracy is much more important than the first because it leads to a different amplitude of the parallax vector, rather than just a different direction. That is, the amplitude of $\pi_{\mathrm{E}}$ in Equation (13) is the same for the two solutions $\Delta u_{0,-, \pm}$ or for the two solutions $\Delta u_{0,+, \pm}$, but is not the same between these two pairs. Because it is only the amplitude of the parallax vector that enters the lens mass and distance, degeneracies in solutions that affect only the direction of $\boldsymbol{\pi}_{\mathrm{E}}$ are relatively unimportant.

As pointed out by Gould \& Horne (2013), the presence of a planet can resolve the second (amplitude) degeneracy. If the planetary caustic appears in both light curves, then this can prove, for example, that the source trajectory appeared on the same side of the lens for the two observatories. This turns out to be the situation here.

Nevertheless, the first degeneracy $\left( \pm u_{0}\right)$ does persist. The geometries of the two solutions are illustrated in Figure 4, and the parameter values are listed in Tables 1 and 2. Note that the $u_{0}<0$ solution is disfavored by $\chi^{2}$, but is not completely excluded.

\section{TWO TESTS OF EARTH-ORBIT-BASED MICROLENSING PARALLAX}

\subsection{Fit to Ground Based Data of OGLE-2014-BLG-0124}

The Einstein timescale of this event was unusually long, $t_{\mathrm{E}}=$ 150 days. Such events very often yield parallax measurements, particularly when the parallax is relatively large and the source is relatively bright, such as in the present case. It is therefore useful to check the parallax measurement that can be made from just ground-based data for two reasons. First, we would like to quantify the improvement that is achieved by incorporating Spitzer data. Second, we would like to check whether groundbased parallaxes (which rely on subtle light curve effects that are potentially corrupted by systematics) agree with a very robust independent determination. In fact, of the dozens of microlens parallax measurements that have been made (from a total of $>10^{4}$ events), there has been only one completely rigorous test and one other quite secure test (Section 6.2).

We repeat the same procedures described in Sections 4 and 5 except that we exclude Spitzer data. We report the results in 
Table 3

$\mu$ lens Parameters (OGLE Only)

\begin{tabular}{|c|c|c|c|}
\hline Parameter & Unit & $u_{0}>0$ & $u_{0}<0$ \\
\hline \multirow[t]{2}{*}{$\chi^{2} /$ dof } & & 6621 & 6622 \\
\hline & & $/ 6732$ & $/ 6732$ \\
\hline \multirow[t]{2}{*}{$t_{0}-6800$} & day & 36.170 & 36.182 \\
\hline & & 0.051 & 0.054 \\
\hline \multirow[t]{2}{*}{$u_{0}$} & & 0.2099 & -0.1964 \\
\hline & & 0.0197 & 0.0201 \\
\hline \multirow[t]{2}{*}{$t_{\mathrm{E}}$} & day & 131.1 & 140.7 \\
\hline & & 9.7 & 13.1 \\
\hline \multirow[t]{2}{*}{$s$} & & 0.9260 & 0.9366 \\
\hline & & 0.0096 & 0.0097 \\
\hline \multirow[t]{2}{*}{$q$} & $10^{-3}$ & 0.752 & 0.696 \\
\hline & & 0.092 & 0.086 \\
\hline \multirow[t]{2}{*}{$\alpha$} & deg & 78.514 & -78.566 \\
\hline & & 0.183 & 0.168 \\
\hline \multirow[t]{2}{*}{$\rho$} & $10^{-3}$ & 1.60 & 1.36 \\
\hline & & 0.46 & 0.44 \\
\hline \multirow[t]{2}{*}{$\pi_{\mathrm{E}, \mathrm{N}}$} & & 0.0179 & -0.0356 \\
\hline & & 0.0122 & 0.0443 \\
\hline \multirow[t]{2}{*}{$\pi_{\mathrm{E}, \mathrm{E}}$} & & 0.1077 & 0.1251 \\
\hline & & 0.0233 & 0.0247 \\
\hline \multirow{2}{*}{$\gamma_{\|}$} & $\mathrm{yr}^{-1}$ & -0.148 & -0.138 \\
\hline & & 0.023 & 0.023 \\
\hline \multirow[t]{2}{*}{$\gamma_{\perp}$} & $\mathrm{yr}^{-1}$ & 0.62 & -0.52 \\
\hline & & 0.68 & 0.67 \\
\hline
\end{tabular}

Table 4

Physical Parameters (OGLE Only)

\begin{tabular}{|c|c|c|c|}
\hline Parameter & Unit & $u_{0}>0$ & $u_{0}<0$ \\
\hline \multirow[t]{2}{*}{$M_{\text {host }}$} & $M_{\odot}$ & 0.81 & 0.74 \\
\hline & & 0.20 & 0.21 \\
\hline \multirow{2}{*}{$M_{\text {planet }}$} & $M_{\text {jup }}$ & 0.63 & 0.53 \\
\hline & & 0.18 & 0.16 \\
\hline \multirow[t]{2}{*}{ Distance } & $\mathrm{kpc}$ & 4.92 & 4.25 \\
\hline & & 0.69 & 0.72 \\
\hline \multirow{2}{*}{$a_{\perp}$} & $\mathrm{AU}$ & 3.16 & 3.13 \\
\hline & & 0.46 & 0.47 \\
\hline \multirow[t]{2}{*}{$\tilde{v}_{\mathrm{N}, \text { hel }}$} & $\mathrm{km} / \mathrm{s}$ & 28.9 & -28.0 \\
\hline & & 38.6 & 30.5 \\
\hline \multirow[t]{2}{*}{$\tilde{v}_{\mathrm{E}, \mathrm{hel}}$} & $\mathrm{km} / \mathrm{s}$ & 149.6 & 111.5 \\
\hline & & 27.5 & 17.2 \\
\hline \multirow[t]{2}{*}{$\mu_{\text {hel }}$} & $\mathrm{mas} / \mathrm{yr}$ & 2.48 & 2.81 \\
\hline & & 0.64 & 0.86 \\
\hline \multirow[t]{2}{*}{$\beta=\left(E_{\mathrm{kin}} / E_{\mathrm{pot}}\right)_{\perp}$} & & 0.40 & 0.38 \\
\hline & & 0.31 & 0.30 \\
\hline \multirow[t]{2}{*}{$\theta_{\mathrm{E}}$} & mas & 0.72 & 0.83 \\
\hline & & 0.19 & 0.26 \\
\hline
\end{tabular}

Tables 3 and 4 . Figure 5 compares the constraints on the parallax vector from the OGLE data alone and the joint fit to the OGLE and Spitzer data.

The first point is that with only OGLE data, the $u_{0}>0$ and $u_{0}<0$ solutions are statistically indistinguishable (the degeneracy of direction). For the dominant east component, these yield $\pi_{\mathrm{E}, E}=0.108 \pm 0.023$ and $0.125 \pm 0.025$, i.e.,

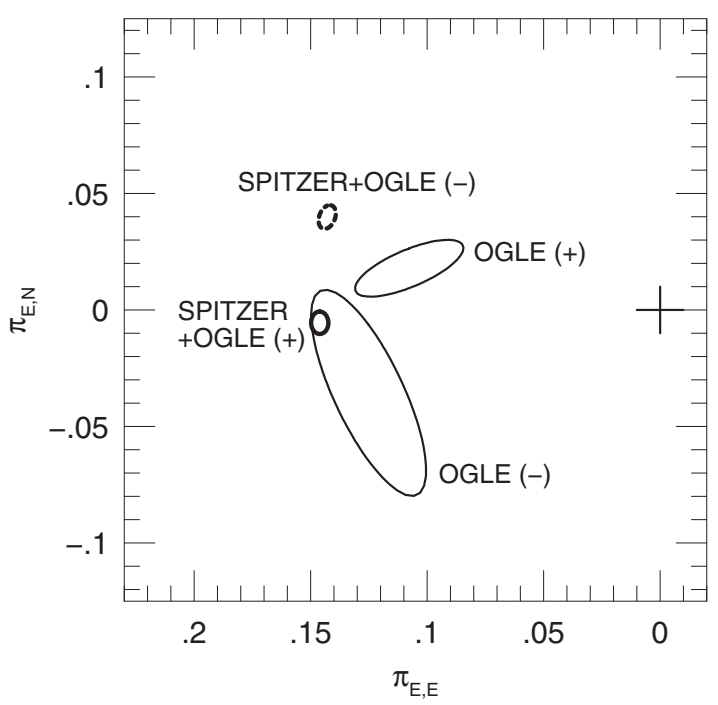

Figure 5. Error contours $\left(\Delta \chi^{2}=1\right)$ in the $\pi_{\mathrm{E}}$ plane for two solutions $\left(u_{0}>0\right.$ and $u_{0}<0$ ) for each of two cases (OGLE only and Spitzer+OGLE), shown in standard and bold curves, respectively. For Spitzer+OGLE, the $u_{0}<0$ solution is displayed as a dashed curve as a reminder that this solution is disfavored but not formally excluded $\left(\Delta \chi^{2}=7\right)$. By contrast, the OGLE-only solutions differ by $\Delta \chi^{2}=1$. The $u_{0}>0$ and $u_{0}<$ solutions are indicated by $(+)$ and $(-)$, respectively.

$21 \%$ and $20 \%$ errors, respectively. Since both solutions must be considered viable, we should adopt $\pi_{\mathrm{E}}=(-0.009,0.116) \pm$ $(0.039,0.026)$ as the "prediction" of the OGLE data.

Second, in contrast to many past parallax measurements, which are typically much more constraining in the direction of Earth's acceleration (east), this measurement has comparable errors in the north and east directions. This is undoubtedly due to the very long $t_{\mathrm{E}}$, since analyses by Gould et al. (1994), Smith et al. (2003), and Gould (2004), all show that so-called "onedimensional parallaxes" explicitly arise from the shortness of events relative to a year.

Third, the ground-based parallax measurements are off by $0.1 \sigma$ and $1.1 \sigma$ respectively. The probability for this level of discrepancy, assuming purely statistical errors, is $\sim 54 \%$, i.e., quite consistent.

Fourth, including Spitzer data improves the precision by a factor of seven in the east direction and a factor of eight in the north direction. This demonstrates the tremendous power of space-based parallaxes relative to the ground, even for an event whose characteristics make it especially favorable for groundbased measurement.

Finally, we note that while the parallax measurements with and without Spitzer data are consistent at the $1 \sigma$ level using the OGLE-only error bar, the derived lens mass and distance both show much closer agreement relative to their statistical errors. This is because the errors in both quantities are dominated by the errors in $\rho$ (through $\theta_{\mathrm{E}}$ ) and this quantity is poorly determined in the present case.

\subsection{A Second Direct Test: MACHO-LMC-5}

Spitzer observations of OGLE-2014-BLG-0124 provide only the second direct test of a microlens parallax measurement derived from so-called "orbital parallax," i.e., distortions in the light curve due to the accelerated motion of Earth. Such tests are quite important because microlens parallaxes are derived from very subtle deviations in the light curve, which could potentially be corrupted by —or be even entirely caused by—instrumental 
systematics and/or real physical processes unrelated to Earth's motion.

In the present case, we found that the accuracy of the ground based measurement of "orbital parallax" (as judged by the comparison to the much more precise Earth-Spitzer measurement) was nearly as good as the relatively small formal errors of $\sigma\left(\boldsymbol{\pi}_{\mathrm{E}}\right)=(0.039,0.026)$.

The only other event for which data exist to directly test a microlens parallax measurement is MACHO-LMC-5, which was one of the first microlensing events ever observed. In fact, although these data permit a full two-dimensional test of the parallax measurement, all three papers that addressed this issue (Alcock et al. 2001; Gould 2004; Gould et al. 2004) considered only a one-dimensional test, namely, a comparison of the direction of $\boldsymbol{\pi}_{\mathrm{E} \text {,hel }}$ measured from the microlensing light curve with the direction of $\boldsymbol{\mu}_{\text {hel }}$ measured from Hubble Space Telescope (HST) astrometry. What makes a two-dimensional test possible is the astrometric measurement of $\pi_{\text {rel }}$ (in additional to $\boldsymbol{\mu}_{\text {hel }}$ ), which was available to Gould et al. (2004) (but not the other two papers) via the work of Drake et al. (2004). Then one can directly compare

$$
\tilde{\mathbf{v}}_{\text {hel }, h s t}=\mu_{\text {hel }} \frac{\mathrm{AU}}{\pi_{\text {rel }}} ; \quad \tilde{\mathbf{v}}_{\text {hel }, \mu \text { lens }}=\frac{\pi_{\mathrm{E}, \mathrm{geo}} \mathrm{AU}}{\pi_{\mathrm{E}}^{2} t_{\mathrm{E}}}+\mathbf{v}_{\oplus, \perp} .
$$

We adopt the data set finally assembled by Gould et al. (2004) and find

$$
\begin{aligned}
\tilde{\mathbf{v}}_{\text {hel }, h s t} & =\left[(-32.5,46.73) \mathrm{km} \mathrm{s}^{-1}\right] \times(1 \pm 0.10) \\
\tilde{\mathbf{v}}_{\text {hel }, \mu \text { lens }} & =(-33.8 \pm 6.3,37.0 \pm 1.9) \mathrm{km} \mathrm{s}^{-1}
\end{aligned}
$$

The form of the first equation reflects that the errors are almost perfectly correlated (correlation coefficients $\rho=0.9997$ ) so that the errors can affect the magnitude but not the direction of this vector. By contrast, the errors in the second equation are almost perfectly independent $(\rho=0.015)$. The $\Delta \chi^{2}=1$ error ellipses are shown in Figure 6. In the lower panel, we show the error ellipse predicted for the difference of the two measurements (based on the sum of the covariance matrices) compared to the actual difference in Equation (15). This yields $\chi^{2}=2.87$ for two degrees of freedom, which has a probability $\exp \left(-\chi^{2} / 2\right)=24 \%$, i.e., quite consistent.

In addition to this direct test, there is one previous indirect test. For the case of the two-planet system OGLE-2006-BLG109Lb,c, the mass and distance derived from a combination of microlens parallax and finite source effects were $M=$ $\theta_{\mathrm{E}} / \kappa \pi_{\mathrm{E}}=0.51 \pm 0.05 M_{\odot}$ and $D_{L}=1.49 \pm 0.12 \mathrm{kpc}$. (Gaudi et al. 2008; Bennett et al. 2010). These predict a dereddened source flux of $H_{0}=M_{H}+5 \log \left(D_{L} / 10 \mathrm{pc}\right)=5.94+10.87=$ 16.81. From high-resolution Keck imaging, Bennett et al. (2010) found $H=17.09 \pm 0.20$. They estimated an extinction of $A_{H}=0.3 \pm 0.2$. Hence, the two estimates of $H$ differ by $\Delta H=0.01 \pm 0.28$, not accounting for intrinsic dispersion in $M_{H}$ as a function of mass.

\section{DISCUSSION}

The projected velocity $\tilde{\mathbf{v}}$ is both the most precisely and most robustly measured physical parameter, but it is also the most puzzling. Recall from Section 3 that $\tilde{\mathbf{v}}_{\text {hel }}=(0,105) \mathrm{km} \mathrm{s}^{-1}$ can be derived from direct inspection of the light curve, values that are confirmed and measured to a precision of $3 \mathrm{~km} \mathrm{~s}^{-1}$ by the light curve analysis as summarized in Table 2.
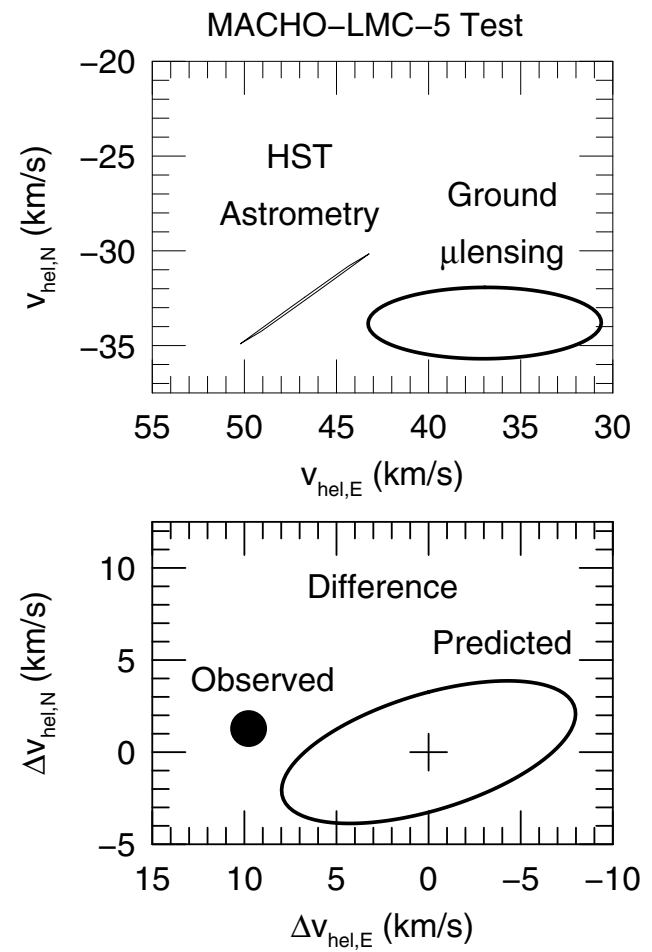

Figure 6. Upper panel: comparison of projected velocity $\tilde{\mathbf{v}}$ as determined from microlensing light curve and HST astrometry for MACHO-LMC-5, which was discovered by the MACHO group in 1993. Lower panel: predicted difference (zero with error ellipse) between these two measurements compared to observed difference. The $\Delta \chi^{2}=2.87$ (for 2 dof) implies consistency at the $24 \%$ level. MACHO-LMC-5 is the only ground-based parallax measurement (other than OGLE-2014-BLG-0124) for which such a rigorous test is possible. Both events pass this test.

From the magnitude $\tilde{v}_{\text {hel }} \sim 100 \mathrm{~km} \mathrm{~s}^{-1}$, one would conclude that the lens is most likely at intermediate distance in the disk. This is because

$$
\mu=\frac{\tilde{v}}{\mathrm{AU}} \pi_{\mathrm{rel}} .
$$

Hence, for stars within 1-2 kpc of the Sun ( $\operatorname{so} \pi_{\text {rel }} \sim \pi_{l}$ ), we have $\tilde{v}_{\text {hel }} \sim v_{\perp, \text { rel }}$, i.e., the transverse velocity of the star in the frame of the Sun. Since very few stars are moving at $\sim 100 \mathrm{~km} \mathrm{~s}^{-1}$, it is unlikely that a nearby star would have $\tilde{v}_{\text {hel }} \sim 100 \mathrm{~km} \mathrm{~s}^{-1}$. By the same token, bulge lenses have $\pi_{\text {rel }} \lesssim 0.03$, meaning that this projected velocity measurement would correspond to $\mu_{\text {geo }} \lesssim 0.5$ mas yr $^{-1}$. Since typical values for bulge lenses are $\mu_{\text {geo }} \sim 4$ mas yr $^{-1}$ and since the probability of slow lenses scales $\propto \mu_{\text {geo }}^{2}$, bulge lenses with this projected velocity are also unlikely. Hence, the estimate $D_{l}=4.1 \pm 0.6 \mathrm{kpc}$ from Table 2 seems at first sight quite consistent with these general arguments.

The problem is that the direction of $\tilde{\mathbf{v}}_{\text {hel }}$, almost due east, is quite unexpected for disk lenses at intermediate distance. The fact that the Sun and the lens both partake of the Galaxy's flat rotation curve, while the bulge sources have roughly isotropic proper motions implies that the mean heliocentric relative proper motion should be $\left\langle\boldsymbol{\mu}_{\mathrm{hel}}\right\rangle=\boldsymbol{\mu}_{\mathrm{SgrA} *}=(5.5,3.2) \mathrm{mas} \mathrm{yr}^{-1}$. Hence, for an assumed distance of $D_{l}=4.1 \mathrm{kpc}\left(\pi_{\mathrm{rel}}=0.12 \mathrm{mas}\right)$, there is an offset

$$
\Delta \boldsymbol{\mu}_{\text {hel }}=\mu_{\text {hel }}-\left\langle\mu_{\text {hel }}\right\rangle=(-5.6,0.5) \text { mas yr }^{-1} .
$$

While it is not impossible that the source star is responsible for this motion (although it is relatively large considering that the one-dimensional dispersion of bulge lenses is $\sigma_{\mu} \sim 3 \mathrm{mas} \mathrm{yr}^{-1}$ ) 
or that there is some contribution from the peculiar motion of the lens itself, the problem is that this unusually large motion just happens to be of just the right size and direction to push $\tilde{v}_{N} \sim 0$. Of course, the lens must be going in some direction, but east is a very special direction in the problem because that is the projected direction of the Earth-Spitzer axis.

One generic way to produce a spurious alignment between the inferred direction of lens motion and the Earth-Spitzer axis is to introduce "noise" in the sparse epochs of the early Spitzer light curve. We do not expect instrumental noise at this level and do not see any evidence of it in the late Spitzer light curve. However, one way to introduce astrophysical "noise" would be to assume that the true direction of motion was very different and that the planetary deviation seen in the Spitzer light curve was from a second unrelated planet. This would require some fine-tuning because fitting even four to five deviated points to an already-determined lens geometry is not trivial. However, there is a stronger argument against this scenario: the ground-based data by themselves predict the same general trajectory (albeit with seven times larger errors), so that even without having seen the Spitzer data, one would predict that Spitzer would see deviations due to the ground-observed planet at approximately this epoch.

Hence, we conclude that while the alignment of $\tilde{\mathbf{v}}_{\text {hel }}$ with the Earth-Spitzer axis is indeed a puzzling coincidence, there are no candidate explanations for this other than chance alignment.

\section{CONCLUSIONS}

OGLE-2014-BLG-0124 is the first planetary microlensing event with a space-based measurement of the vector microlens parallax $\boldsymbol{\pi}_{\mathrm{E}}$. Combining $\pi_{\mathrm{E}}$ and $\theta_{\mathrm{E}}$ provides a means to precisely measure masses of the host star and planet in microlensing events. In most planetary microlensing events, $\pi_{\mathrm{E}}$ is the limiting factor in obtaining a direct measurement of the planet's mass (but see Zhu et al. 2014). In this case, the combination of data from both OGLE and Spitzer gives an error in the amplitude of the parallax that is only $2.5 \%$, implying that it contributes negligibly to the uncertainty in the host mass $M=\theta_{\mathrm{E}} / \kappa \pi_{\mathrm{E}}=0.71 \pm$ $0.22 M_{\odot}$. Rather, in contrast to the great majority of planetary microlensing events discovered to date, this uncertainty is dominated by the error in $\theta_{\mathrm{E}}$. That is, whereas most current planetary events have caustic crossings that yield a precise measurement of $\rho=\theta_{*} / \theta_{\mathrm{E}}$, so that the fractional error in $\theta_{\mathrm{E}}$ is just that of $\theta_{*}$ (typically $\sim 7 \%$ ), OGLE-2014-BLG-0124 did not undergo caustic crossings. Rather, there is an upper limit on $\rho$ because if it were too big, the source would have approached close enough to a cusp to give rise to detectable effects, and a lower limit because small $\rho$ implies large $\theta_{\mathrm{E}}=\theta_{*} / \rho$ and thus large mass and large lens-source relative parallax $\pi_{\text {rel }}=\theta_{\mathrm{E}} \pi_{\mathrm{E}}$. The combination would make the lens bright enough to be seen for $\rho<6.5 \times 10^{-4}$. Hence the mass of the planet is $m=0.51 \pm 0.16 M_{\text {jup }}$ and its projected separation is $a_{\perp}=3.1 \pm 0.5 \mathrm{AU}$. It lies at a distance $D_{L}=4.1 \pm 0.6 \mathrm{kpc}$ from the Sun.

The high precision of the Earth-Spitzer microlens parallax allows the first rigorous test of a ground-based $\boldsymbol{\pi}_{\mathrm{E}}$ measurement from OGLE-only data, which yielded a $22 \%$ measurement of
$\pi_{\mathrm{E}}$. The Spitzer data show that this measurement is correct to within $1.1 \sigma$. We use archival data to construct a second test using purely astrometric HST data to confirm the twodimensional vector projected velocity $\tilde{\mathbf{v}}$ for MACHO-LMC-5 that was derived from the microlensing data. These tests show that ground-based microlensing parallaxes are reliable within their stated errors in the relatively rare cases that they can be measured.

The OGLE project has received funding from the European Research Council under the European Community's Seventh Framework Programme (FP7/2007-2013) / ERC grant agreement No. 246678 to A.U. Work by J.C.Y. was performed under contract with the California Institute of Technology (Caltech)/ Jet Propulsion Laboratory (JPL) funded by NASA through the Sagan Fellowship Program executed by the NASA Exoplanet Science Institute. A.G. was supported by NSF grant AST 1103471 and NASA grant NNX12AB99G. Work by J.C.Y., A.G., and S.C. was supported by JPL grant 1500811 . Work by C.H. was supported by the Creative Research Initiative Program (2009-0081561) of the National Research Foundation of Korea. This work is based in part on observations made with the Spitzer Space Telescope, which is operated by the Jet Propulsion Laboratory, California Institute of Technology under a contract with NASA.

\section{REFERENCES}

Alcock, C., Allsman, R. A., Alves, D. R., et al. 2001, Natur, 414, 617 Bennett, D. P., Rhie, S. H., Nikolaev, S., et al. 2010, ApJ, 713, 837 Bensby, T., Yee, J. C., Feltzing, S., et al. 2013, A\&A, 549A, 147

Bessell, M. S., \& Brett, J. M. 1988, PASP, 100, 1134

Claret, A. 2000, A\&A, 363, 1081

Dong, S., Gould, A., Udalski, A., et al. 2009, ApJ, 695, 970

Dong, S., Udalski, A., Gould, A., et al. 2007, ApJ, 664, 862

Drake, A. J., Cook, K. H., \& Keller, S. C. 2004, ApJ, 607, L29

Gaudi, B. S. 2012, ARA\&A, 50, 411

Gaudi, B. S., Bennett, D. P., Udalski, A., et al. 2008, Sci, 319, 927

Gould, A. 1994, ApJL, 421, L71

Gould, A. 1999, ApJ, 514, 869

Gould, A. 2000a, ApJ, 535, 928

Gould, A. 2000b, ApJ, 542, 785

Gould, A. 2004, ApJ, 606, 319

Gould, A. 2008, ApJ, 681, 1593

Gould, A, Bennett, D. P., \& Alves, D. R. 2004, ApJ, 614, 404

Gould, A., \& Gaucherel, C. 1997, ApJ, 477, 580

Gould, A., \& Horne, K. 2013, ApJ, 779, 28

Gould, A., \& Loeb, A. 1992, ApJ, 396, 104

Gould, A., Miralda-Escudé, J., \& Bahcall, J. N. 1994, ApJL, 423, L105

Han, C. 2006, ApJ, 638, 1080

Kervella, P., Thévenin, F., Di Folco, E., \& Ségransan, D. 2004, A\&A, 426, 297

Nataf, D. M., Gould, A., Fouqué, P., et al. 2013, ApJ, 769, 88

Pejcha, O., \& Heyrovský, D. 2009, ApJ, 690, 1772

Poleski, R., Udalski, A., Dong, S., et al. 2014, ApJ, 782, 47

Refsdal, S. 1966, MNRAS, 134, 315

Schechter, P. L., Mateo, M., \& Saha, A. 1993, PASP, 105, 1342

Skowron, J., Udalski, A., Gould, A., et al. 2011, ApJ, 738, 87

Smith, M., Mao, S., \& Paczyński, B. 2003, MNRAS, 339, 925

Udalski, A. 2003, AcA, 53, 291

Udalski, A., Szymanski, M., Kaluzny, J., et al. 1994, AcA, 44, 227

Yee, J. C., Svartzvald, Y., Gal-Yam, A., et al. 2012, ApJ, 755, 102

Yoo, J., DePoy, D. L., Gal-Yam, A., et al. 2004, ApJ, 603, 139

Zhu, W., Penny, M., Mao, S., Gould, A., \& Gendron, R. 2014, ApJ, 788, 73 